\title{
This Issue of the Journal of Urban Health
}

David H. Frankel and David Vlahov

"For better or worse," in both public health and the practice of medicine, neither data nor apparent fact take the place of human experience and interpretation. We often rely on distant eyes to lead us in even our own professional neighborhood.

So, in this spirit, the Journal begins this issue with a new section, "The Literature of Public Health." It is meant to inform our readers by calling on columnists and researchers from around the world to tell us just what they make of current events and what we should make of the current literature affecting urban health. We will expand the section to include columnists from far-flung parts of the globe and literature reviewers from many additional disciplines. We want this to be useful to your practice. Tell us what you think. 